\title{
2 Reform der Notfallversorgung
}

\author{
Michael Slowik und Kerstin Bockhorst
}

Die Versorgung von Menschen in lebensbedrohlichen medizinischen Notfallsituationen sowie von nicht lebensbedrohlich erkrankten Patientinnen und Patienten mit dringlichem Behandlungsbedarf wird gegenwärtig über fünf Säulen organisiert:

1. die ambulante Versorgung in den Sprechzeiten der niedergelassenen Ärzte,

2. den kassenärztlichen Bereitschaftsdienst außerhalb der Sprechstundenzeiten niedergelassener Ärzte (Notdienst),

3. das Rettungswesen,

4. die Notaufnahmen der Krankenhäuser als durchgehend geöffnete Ambulanzen und

5. die stationäre Notfallversorgung.

Diese fünf Säulen nehmen unterschiedliche Funktionen und Aufgaben in der ambulanten und stationären Notfallversorgung wahr und unterliegen sowohl rechtlich als auch organisatorisch unterschiedlichen, kaum aufeinander abgestimmten Rahmenbedingungen. Die (digitale) Kommunikation zwischen den Versorgungsbereichen und die planerische Abstimmung sind qualitativ unzureichend oder nicht vorhanden.

Die stationäre Notfallversorgung wurde mit einem Auftrag an den Gemeinsamen Bundesausschuss (G-BA) zur Erarbeitung eines gestuften Systems der Notfallstrukturen in Krankenhäusern durch das Krankenhausstrukturgesetz (KHSG) im Jahr 2016 adressiert. Durch den Beschluss im Jahr 2018 wurden erstmals Mindeststandards für die Teilnahme an der stationären Notfallversorgung bundeseinheitlich definiert (\$ 136c Abs. 4 SGB V). Unberührt von den Notfallstufen-Regelungen des G-BA bleibt die Versorgung von ambulanten Notfallpatientinnen und -patienten. Es fehlt ein bundesweiter Überblick 
dazu, wo, wann und in welchem Umfang Vertragsärzte und Krankenhäuser ambulante Notfallversorgung leisten. Zudem werden die Standorte und Kapazitäten der stationären und der ambulanten Notfallversorger aktuell nicht einheitlich und gemeinsam geplant.

Aus Patientenperspektive trägt die fehlende Abstimmung der Bereiche dazu bei, dass Patientinnen und Patienten nicht immer in der angemessenen Versorgungsebene behandelt werden. Patientinnen und Patienten, die ambulant behandelt werden könnten, werden in den Notaufnahmen der Krankenhäuser oder häufig sogar stationär versorgt. Aktuell besteht weder eine organisatorische noch eine digitale Verknüpfung zwischen den Leitstellen des Rettungsdienstes und den Leitstellen des KV-Bereitschaftsdienstes. Dies hat zur Folge, dass die Tatsache, welche Notfallnummer die Patientin bzw. der Patient wählt (112 oder 116 117), über die Versorgungsebene entscheidet und nicht der Versorgungsbedarf das ausschlaggebende Kriterium ist.

Ähnlich sieht es mit Blick auf die Kommunikation zwischen Rettungsdienst und Krankenhaus aus. Die Kenntnis des Rettungsdienstes über die Ausstattung und Ressourcen der Krankenhäuser endet häufig an der Kreisgrenze. Auch hier besteht großer Bedarf an einer besseren Kommunikation und digitalen Vernetzung über Kreis- und Ländergrenzen hinweg.

\subsection{Neustrukturierung der stationären Notfallversorgung}

Das im April 2018 vom G-BA beschlossene stationäre Notfallstufenkonzept ist vom Gesetzgeber streng sektoral angelegt und sieht noch keine Verknüpfung zu ambulanten Notfallstrukturen vor. Eigentlicher Anlass des Regelungsauftrages an den G-BA durch das KHSG war der finanzielle Ausgleich von unterschiedlich hohen Vorhaltekosten für die Notfallversorgung, die das DRG-System aufgrund seines Fallbezugs nicht abbilden kann. De facto werden durch die Notfallstufen auch Mindeststandards für die Teilnahme an der Notfallversorgung und Qualitätsanforderungen geschaffen.

\subsubsection{Hintergrund und gesetzlicher Auftrag}

Stationäre Notfälle wurden in Deutschland vor Einführung der Notfallzuschläge ausschließlich über das DRG-Fallpauschalensystem finanziert. Krankenhäuser mit vielen Notfällen erlösen viele fallbezogene Entgelte und können ihre Strukturen dadurch selbsttragend finanzieren. Krankenhäuser, die trotz vorgehaltener Strukturen wenige Patientenkontakte haben, generieren hingegen keine ausreichenden Erlöse zur Finanzierung ihrer Notfallstrukturen. Es entsteht ein ungewollter Anreiz zum Rückzug aus der Rund-um-die-Uhr-Notfallversorgung, denn Krankenhäuser ohne Notfallvorhaltungen haben einen Vorteil in diesem Finanzierungssystem und sind besser ausgelastet. Um diesen ungewollten Vorteil auszugleichen, wurde bereits vor Inkrafttreten des KHSG in der stationären Notfallversorgung zwischen einer Teilnahme und einer Nichtteilnahme an der Notfallversorgung unterschieden. Mit Krankenhäusern, die nicht an der Notfallversorgung teilnehmen, war ein Abschlag in 
Höhe von 50 Euro je vollstationären Fall zu vereinbaren. Die Umsetzung dieser Regelung wurde in den Ländern jedoch sehr unterschiedlich gelebt. Der dringend notwendige Ausgleich der Vorhaltekosten erfolgte aufgrund der unvollständigen Umsetzung der Abschlagsregelung und fehlender Mindestanforderungen an eine Notfallteilnahme in vielen Fällen nicht.

Aus den zuvor genannten Gründen hatte der G-BA mit dem KHSG den Auftrag erhalten, die Notfallversorgung neu zu strukturieren und ein gestuftes System der Notfallstrukturen in Krankenhäusern zu beschließen. Krankenhäuser sollen, gemessen am Umfang ihrer Vorhaltungen für die Notfallversorgung, einer Stufe zugeordnet werden. Dem G-BA wurde daher aufgetragen, für jede Notfallstufe strukturelle Mindestanforderungen für die folgenden Kriterien zu definieren.

Kriterien für strukturelle Mindestanforderungen der Notfallstufen

- Art und Anzahl von Fachabteilungen

- Anzahl und Qualifikation des vorzuhaltenden Fachpersonals

- Kapazitäten zur Versorgung von Intensivpatientinnen und -patienten

- medizinisch-technische Ausstattung

- Strukturen und Prozesse der Notfallaufnahme

Auf Grundlage der Stufenzuordnung sollen Krankenhäuser der Höhe nach gestaffelte Zuschläge für ihre Teilnahme an der Notfallversorgung erhalten. Krankenhäuser, welche die strukturellen Mindestanforderungen nicht erfüllen, müssen Abschläge hinnehmen.

\subsubsection{Leitgedanken zur Differenzierung der Notfallstufen}

Entsprechend dem Gesetzesauftrag hat der G-BA differenziert für jede Notfallstufe strukturelle Mindestanforderungen festlegt, um Krankenhäuser zukünftig gemessen am Umfang ihrer Vorhaltungen für die Notfallversorgung einer Stufe zuordnen zu können. Die Mindestanforderungen sind zu jeder Zeit am Standort des Krankenhauses zu erfüllen. Krankenhäuser, die z.B. nachts oder am Wochenende keine Notfallpatientinnen und -patienten aufnehmen, erfüllen nicht die Anforderungen der Notfallstufenregelungen.

Das Stufenmodell des G-BA unterscheidet drei zuschlagsfähige Notfallstufen, eine Stufe ohne Zu- und Abschläge und eine Stufe der Nichtteilnahme an der Notfallversorgung, für die verbindliche Abschläge vorzusehen sind (s. Abb. 1). Je mehr notfallrelevante Fachabteilungen ein Krankenhaus am Standort rund um die Uhr vorhält, umso höher ist auch der finanzielle Aufwand der Vorhaltung, der durch die Einsortierung in die Notfallstufen abgebildet wird. Während ein Basisnotfallversorger die Fachabteilungen Chirurgie oder Unfallchirurgie und Innere Medizin vorhält, zeichnen sich die Notfallversorger der erweiterten 


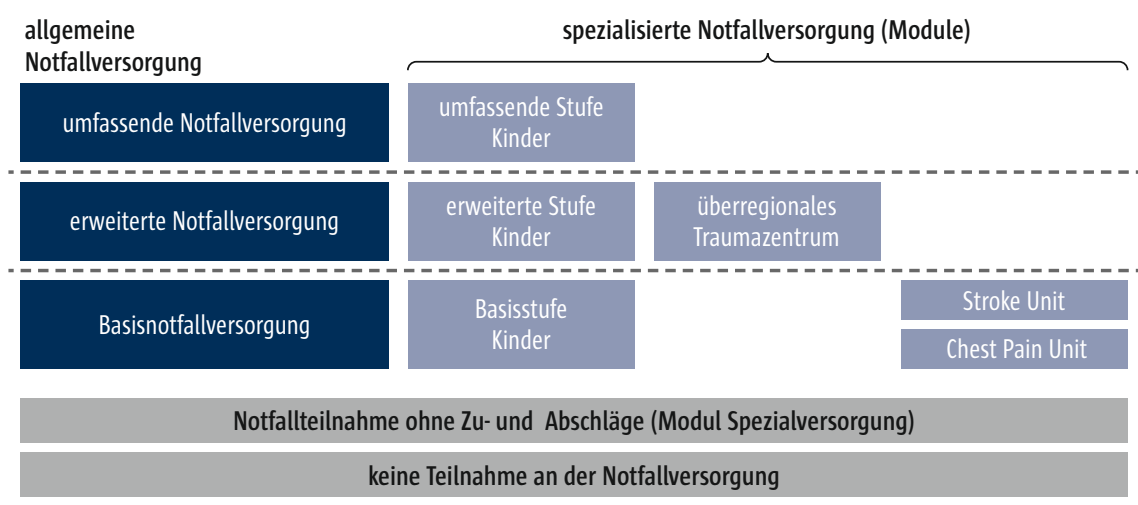

Abb. 1 Stationäres Notfallstufenkonzept gemäß Beschluss des Gemeinsamen Bundesausschusses vom 19. April 2018

und der umfassenden Stufe dadurch aus, dass sie darüber hinaus weitere, nach Notfallrelevanz abgestufte Fachabteilungen vorhalten. Ein Basisnotfallversorger muss in der Lage sein, leichtere Notfälle direkt zu versorgen sowie schwerwiegende Erkrankungen und Verletzungen zu erkennen, um die Patientinnen und Patienten bei Bedarf zielgerichtet an einen Spezialversorger weiterverlegen zu können. Daher gehört u.a. die computertomografische Bildgebung zur Ausstattung eines Basisversorgers. In den höheren Stufen muss die medizintechnische und personelle Ausstattung für die Versorgung von Patientinnen und Patienten mit notfallmedizinisch besonders relevanten Krankheitsbildern, den sog. Tracerdiagnosen, geeignet sein. Hierzu zählen insbesondere die Diagnosen Schädel-Hirn-Trauma, Schlaganfall, Polytrauma, ST-Hebungsinfarkt, plötzlicher Kreislaufstillstand und Sepsis (Fischer et al. 2016). Zudem steigen je Stufe die Anzahl der vorzuhaltenden Intensivbetten und die Anforderungen an die Zuverlegungsmöglichkeiten von Notfallpatientinnen und -patienten.

Da an die Notfallversorgung von Kindern andere Strukturanforderungen zu stellen sind, wird das dreistufige Zuschlagsmodell durch ein ebenfalls dreistufiges Modul „Kindernotfallversorgung“ ergänzt. Weiterhin trägt das Modul „Schwerverletzenversorgung“ der Tatsache Rechnung, dass es sich bei den überregionalen Traumazentren um Spezialversorger mit einem kleinen, aber hochspezialisierten Fachabteilungsangebot handelt. Diese Spezialversorger werden unabhängig von der Breite ihres Fachabteilungsspektrums der Stufe der erweiterten Notfallversorgung zugeordnet. Darüber hinaus gibt es Einrichtungen, die unbestreitbar eine wichtige Rolle in der Notfallversorgung spielen, deren Vorhaltekosten aber bereits zielgerichtet über andere Finanzierungsinstrumente vergütet werden. Hier sind insbesondere Einrichtungen gemeint, die nicht über das DRG-Fallpauschalensystem finanziert werden (z.B. besondere Einrichtungen, psychiatrische Fachkliniken) und deren Vorhaltekosten bereits in Budgets eingepreist sind. Diese Einrichtungen werden vom Abschlag befreit, erhalten allerdings auch keine Zuschläge. 


\subsubsection{Anforderungen an die Zentrale Notaufnahme}

Eine Kernforderung der Krankenkassen war es, dass die Patientin oder der Patient im Notfall eine bekannte, feste Anlaufstelle haben soll und dort interdisziplinär ausgebildetes Personal entscheidet, in welcher Fachrichtung die Patientin oder der Patient richtig aufgehoben ist. Kritisch Erkrankten kann nicht zugemutet werden, eigenständig eine Zuordnung in eine fachgebietsspezifische Notaufnahme vorzunehmen. Dieser Forderung wird in den G-BARegelungen Rechnung getragen, indem der ganz überwiegende Anteil der Notfallpatientinnen und -patienten in einer Zentralen Notaufnahme (ZNA) aufgenommen werden muss und jede Patientin und jeder Patient innerhalb von zehn Minuten nach Eintreffen in der ZNA eine Ersteinschätzung zu ihrer oder seiner Behandlungsdringlichkeit erhält. Das verantwortliche ärztliche und pflegerische Personal hat umfangreiche Erfahrung in der Versorgung von Notfallpatientinnen und -patienten, was durch die Zusatzweiterbildung „Klinische Akut- und Notfallmedizin“ der verantwortlichen Ärztin oder des verantwortlichen Arztes bzw. die Zusatzqualifikation „Notfallpflege“ der verantwortlichen Pflegekraft nachzuweisen ist. Spätestens nach 30 Minuten muss auch eine qualifizierte Fachärztin oder ein qualifizierter Facharzt verfügbar sein. Ab der erweiterten Notfallstufe hat die ZNA eine organisatorisch der Notaufnahme angeschlossene Beobachtungsstation mit mindestens sechs Betten vorzuhalten. Dort sollen Notfallpatientinnen und -patienten verbleiben, bei denen eine zeitlich unter 24 Stunden begrenzte Weiterversorgung erforderlich ist oder die Notwendigkeit einer stationären Aufnahme abgeklärt werden muss, bis der weitere Behandlungsweg medizinisch und organisatorisch geklärt ist.

\subsubsection{Verknüpfung zur ambulanten Notfallversorgung}

Der Gesetzgeber hatte die Notfallstufenregelung auf die stationäre Versorgung beschränkt. Allerdings zeigt sich in Deutschland - wie auch in anderen europäischen Ländern - der Trend, dass auch die ambulante Notfallversorgung zunehmend am Krankenhaus stattfindet (Dräther u. Schäfer 2017; Slowik et al. 2018; Baier et al. 2019). Die G-BA-Regelungen appellieren daher an die Notfallkrankenhäuser, eine Kooperationsvereinbarung mit der jeweils zuständigen Kassenärztlichen Vereinigung (KV) über die Versorgung ambulanter Notfälle zu schließen. Dies kann z.B. die Einrichtung einer KV-Notdienstpraxis im oder am Krankenhaus sein.

\subsubsection{Aktuelles Einstufungsverfahren}

In der Notfallstufenvergütungsvereinbarung wurde festgelegt, dass die Vertragsparteien vor Ort jeweils für den Vereinbarungszeitraum die Erfüllung der Mindestvoraussetzungen, z.B. anhand einer Checkliste und ggf. den Ergebnissen etwaiger Qualitätskontrollen des Medizinischen Dienstes gemäß $\$ 275 \mathrm{a}$ 
Abs. 2 S. 3 Nr. 1 SGB V für eine Teilnahme an der Notfallversorgung für den jeweiligen Krankenhausstandort prüfen. Die Festlegung, in welche Notfallstufe bzw. in welches Modul der Krankenhausstandort einzustufen ist, erfolgt verbindlich in der Budgetverhandlung. In den Verhandlungen für das Jahr 2019 erfolgte die erstmalige Festlegung der standortbezogenen Notfallstufen. Auf Basis von ca. 904 vorliegenden Budgetabschlüssen mit geeinten Notfallzu- und -abschlägen wurde deren Verteilung je Budget-IK analysiert. Hierdurch lässt sich eine erste Einschätzung zur stationären Notfallkrankenhausstruktur ableiten.

Gegenwärtig liegen 255 Abschlagsvereinbarungen für Nicht-Teilnehmer in der Budgetdatenbank vor. Darüber hinaus können Planungsbehörden einzelne Standorte als Spezialversorger ausweisen, diese Krankenhäuser sind von $\mathrm{Zu}$ oder Abschlägen ausgenommen und können daher in der Budgetdatenbank nicht identifiziert werden. Bei der Analyse der Notfalleinstufungen zeigt sich eine pyramidale Verteilung der Notfallteilnehmer. Für die Stufe der Basisnotfallversorgung erhalten nach aktuellem Stand 432 Krankenhausstandorte einen Zuschlag. Die Stufe der erweiterten Notfallversorgung inkl. Schwerverletztenversorgung erfüllen 192 Standorte. Zuschläge für die umfassende Notfallversorgung erhalten 114 Standorte. Bei den Notfall-Modulen kann keine Anzahl an Standorten aus den Budgetdaten ermittelt werden, sondern nur die Anzahl an Krankenhäusern (ein Krankenhaus kann somit unter Umständen mehrere Module für mehrere seiner Standorte geeint haben). 41 Krankenhäuser erhalten entweder einen Zuschlag für das Modul Stroke Unit oder das Modul Chest Pain Unit. 217 Krankenhäuser haben Module für die Kindernotfallversorgung vereinbart. Von den 904 Krankenhäusern mit geeinten Notfallzuoder -abschlägen nehmen damit zum 09.10.2020 ca. 72\% an einer zuschlagsfähigen Notfallstufe oder einem Modul teil.

\subsection{Ambulante Notfallstrukturen und Reformbedarf im Rettungswesen}

\subsubsection{Status quo und Handlungsbedarf für bundeseinheitliche und verbindliche Rahmenvorgaben}

Die regionale Gestaltungshoheit und Verantwortung für den kassenärztlichen Bereitschafts- bzw. Notdienst liegt bei den zuständigen KVen, wobei die Umsetzung durch die teilnehmenden Vertragsärztinnen- und ärzte sowie die Krankenhausärztinnen und -ärzte in den Notaufnahmen erfolgt. Die regional unterschiedliche Organisation des kassenärztlichen Bereitschaftsdienstes führt zu einer sehr unübersichtlichen Versorgungssituation für Patientinnen und Patienten. Sie wissen häufig nicht, wann und wo sie bei dringlichem Behandlungsbedarf außerhalb der Sprechstundenzeiten eine Vertragsärztin oder einen Vertragsarzt aufsuchen können. Es gibt oftmals keine festen Notdienstpraxen, stattdessen findet der Notdienst in der Praxis der jeweils diensthabenden Ärz- 
tin oder des Arztes statt. Die Öffnungszeiten der Notdienstpraxen variieren allerdings und decken nicht immer die gesamten sprechstundenfreien Zeiten ab. In einzelnen Ländern (z.B. Berlin) findet die Versorgung außerhalb der Sprechstundenzeiten vorrangig im häuslichen Umfeld der Patientin oder des Patienten durch einen fahrenden ärztlichen Bereitschaftsdienst statt. Angesichts dieser sehr heterogenen Versorgungssituation suchen viele Patientinnen und Patienten direkt die Notaufnahmen der Krankenhäuser auf.

Mit dem Krankenhausstrukturgesetz (KHSC) wurden die KVen verpflichtet, Notdienstpraxen in oder an Krankenhäusern einzurichten, um ambulante und stationäre Notfallstrukturen an bekannter Stelle „unter einem Dach“ zu organisieren. Ziel war es, leicht erkrankte Patientinnen und Patienten ambulant in der KV-Notdienstpraxis zu behandeln, um die Kapazitäten der Notaufnahmen für schwer erkrankte Patientinnen und Patienten frei zu halten. Da die gesetzliche Regelung allerdings keine bundeseinheitlichen Rahmenvorgaben, insbesondere im Hinblick auf die Verfügbarkeit (Öffnungszeiten, regionale Verteilung der Praxen) und die personelle Besetzung der KV-Notdienstpraxen vorsieht, haben sich regional sehr unterschiedliche Angebote entwickelt, die für die Patientinnen und Patienten weiterhin unübersichtlich sind. Es besteht daher große Einigkeit zwischen den verschiedenen Institutionen, die Konzepte zur Reform der Notfallversorgung entwickelt haben, dass es einer Zusammenführung ambulanter und stationärer Notfallstrukturen an zentraler Stelle mit bundeseinheitlichen Rahmenbedingungen bedarf. Offen bleibt allerdings, wie groß der Versorgungsbedarf (Standortanzahl und -auswahl) und der Integrationsgrad für diese Notfallstrukturen ist, um eine gut erreichbare, qualitativ hochwertige und wirtschaftliche Notfallversorgung zu gewährleisten (s. Kap. 2.4.1).

\subsubsection{Rettungsdienst - Status quo und Handlungsbedarf}

Der Rettungsdienst ist historisch bedingt als reine Transportdienstleistung eingestuft und nicht als medizinische Leistung im Sinne des SGB V. Rechtlich sind die Länder für den Rettungsdienst zuständig. Er ist dem Bereich der Gefahrenabwehr zugeordnet und fällt damit in den Kompetenzbereich der Innenministerien. Allerdings steht heute längst nicht mehr der Transport im Vordergrund, sondern die medizinische Erstversorgung, z.B. die Stabilisierung von Vitalfunktionen wie Atmung und Kreislauf an der Einsatzstelle (Gesundheitsberichterstattung des Bundes 1998). Die Koordination der Rettungseinsätze erfolgt über die Leitstellen der Rufnummer 112. Bundesweit gibt es rund 240 dieser Leitstellen, die alle unterschiedlich strukturiert sind. In vielen Fällen sind diese Gesundheitsleitstellen eng mit denen der Feuerwehr verwoben (Wikipedia 2020).

Seit 2012 wird durch die Kassenärztliche Bundesvereinigung (KBV) und die KVen die bundesweit einheitliche Rufnummer 116117 beworben und angeboten, um einen Arzt (Bereitschaftsdienst) auch außerhalb der vertragsärztlichen Sprechstundenzeiten für nicht lebensbedrohliche Notfälle zu erreichen. 
Mit dem Terminservice- und Versorgungsgesetz ist die Bereitschaftsdienstnummer seit 1. Januar 2020 auch rund um die Uhr erreichbar und es erfolgt mithilfe eines softwareunterstützten Fragebogens bereits am Telefon eine Ersteinschätzung zum medizinischen Behandlungsbedarf und zur Wegweisung in die richtige Versorgungsebene. Eine strukturierte Zusammenarbeit mit den Leitstellen der Rufnummer 112 gibt es hingegen nur selten.

Es fehlt an einer digitalen Gesamtstruktur, die alle Akteure der Notfallversorgung (Leitstellen, Rettungsdienst, Krankenhäuser, KV-Bereitschaftsdienst) miteinander vernetzt. Aktuell gibt es weder eine verlässliche Erfassung der Zahl der Rettungsfahrten, noch eine Übersicht, welche Krankenhäuser vom Rettungsdienst angefahren werden und ob die Patientinnen und Patienten dort abschließend versorgt werden können. Für den Rettungsdienst ist es häufig nicht transparent, welche Versorgungsstrukturen an welchen Krankenhäusern angeboten werden. Dies hat zur Folge, dass längst nicht immer der Versorgungsbedarf das ausschlaggebende Kriterium für die Auswahl des anzufahrenden Krankenhauses ist. Insbesondere schwere Notfälle sollten jedoch an wenigen hochspezialisierten Krankenhausstandorten versorgt werden, um die Überlebenschancen der Patientinnen und Patienten zu erhöhen. Nach Angaben der bislang bundesweit einzigen Stelle zur trägerübergreifenden Qualitätssicherung im Rettungsdienst Baden-Württemberg wurde festgestellt, dass über ein Viertel der Patientinnen und Patienten mit Polytrauma nicht primär in ein dafür geeignetes Zentrum transportiert wurde (SQR-BW 2017). Zwischen den Ergebnissen der einzelnen Rettungsdienstbereiche zeigen sich große Unterschiede. Insgesamt lässt sich konstatieren, dass die Qualitätssicherung im Rettungswesen derzeit noch lückenhaft und regional unterschiedlich ausgestaltet ist. Eine Nutzung von Routinedaten findet flächendeckend kaum statt.

\subsection{Politische Forderungen und Gesetzgebungsverfahren zur Reform der Notfallversorgung}

Das Handlungsfeld „Notfallversorgung“ war nicht nur vor der Wahl zum 19. Deutschen Bundestag im Fokus der Interessensvertretungen der Leistungserbringer, Krankenkassen, Fachgesellschaften, Ländervertreter und dem Sachverständigenrat zur Begutachtung der Entwicklung im Gesundheitswesen (SVR). Auch in der aktuellen Regierungszeit und vor der COVID-19-Krise erfolgte eine intensive Diskussion zur Neustrukturierung der Notfallversorgung. Im Juli 2019 veröffentlichte das Bundesministerium für Gesundheit (BMG) einen Diskussionsentwurf eines Gesetzes zur Reform der Notfallversorgung. Beachtenswert war dabei, dass erstmals Empfehlungen und Vorschläge des SVR direkt in ein Gesetzgebungsverfahren übertragen wurden. In Vorbereitung auf die Wahlen zum 19. Deutschen Bundestag und im Zuge der politischen Diskussion um die Neuordnung der Notfallversorgung hatte sich der SVR dazu entschlossen, im Rahmen eines „Werkstattgesprächs“ einen Einblick in seine Reformüberlegungen zur Notfallversorgung zu präsentieren. In 
Anlehnung an Überlegungen aus der Vergangenheit wurde dem Fachpublikum die Idee eines „Integrierten Notfallzentrums“ (INZ) präsentiert. Eine aus niedergelassenen Vertragsärzten und Krankenhäusern betriebene eigenständige Versorgungseinheit soll die Versorgung von ambulanten und ggf. stationären Notfällen übernehmen. Die INZ sollen dabei umfangreich ausgestattet werden und die gesamte notdienstliche Versorgung sektorenübergreifend übernehmen, insbesondere auch den telemedizinischen und den aufsuchenden Bereitschaftsdienst. Vor allem die gemeinsame Organisation von KV und Krankenhaus stellt eine elementare Reformidee dar. Indikationsorientierte Patientensteuerung durch einheitliche Strukturen der ambulanten und teilweise stationären Notfallversorgung wäre die Konsequenz. Mit den umfangreichen Aufgaben wäre auch eine Anpassung des Sicherstellungsauftrags für die notdienstliche Versorgung verbunden. Da die INZ weitergehende Aufgaben übernehmen sollen, wäre der Sicherstellungsauftrag umzugestalten und möglicherweise einem erweiterten Landesausschuss zu übertragen (SVR 2017). Auch die AOK-Gemeinschaft hatte sich ähnlich positioniert und eine umfassende Strukturreform für die Neuordnung der Notfallversorgung befürwortet. Unter anderem wurde gefordert, den Sicherstellungsauftrag einem neu einzurichtenden dreiseitigen Gremium auf Landesebene zu übertragen und den Leistungsumfang der INZ zu erweitern (AOK-BV 2020; Slowik et al. 2018).

Der Diskussionsentwurf des BMG griff die Empfehlungen des SVR auf und skizzierte weitreichende Neuerungen für die Leistungserbringung und Sicherstellung der neu zu gründenden Integrierten Notfallzentren (INZ), die Planung dieser Standorte durch Landesplanungsbehörden und beschrieb weitreichende Reformen im Bereich der Patientensteuerung durch die Einführung gemeinsamer Notfallleitsysteme sowie eine Reform des Rettungswesens. Vor allem die Landesbehörden und Kassenärztlichen Vereinigungen wurden mit umfangreichen Neuerungen konfrontiert, die eine grundlegende Neuregelung des Sicherstellungsauftrags umfassten und neue Kompetenzzuweisungen bei der Planung der INZ und Leitstellen vorsahen. Nach einem Workshop mit den Landesbehörden im Herbst 2019 zeichnete sich eine Überarbeitung des Entwurfs ab, vor allem die Neudefinition des Sicherstellungsauftrags für die ambulante vertragsärztliche Versorgung außerhalb der Sprechstundenzeiten (Notdienst) hatte bei der Standesvertretung der Kassenärzte für großen Widerstand gesorgt. Darüber hinaus wäre eine Neuaufstellung des Rettungswesens nur mit einer Crundgesetzänderung und der erforderlichen Mehrheit im Bundesrat erfolgreich gewesen. Vor allem die Länder lehnten eine zunehmende Verantwortung für die ambulante Notfallversorgung ab und forderten die Aufrechterhaltung der Hoheitsrechte für die Verantwortung des Rettungswesens (Hermann 2019).

Im Januar 2020 veröffentlichte das BMC den Referentenentwurf zur Reform der Notfallversorgung (BMG 2020). Die Kassenärztliche Bundesvereinigung (KBV) begrüßte den Entwurf vor allem mit dem beabsichtigten Ziel, die Angebote der Akut- und Notfallversorgung besser zu verzahnen (KBV 2020). Auch 
im Vorfeld des Gesetzgebungsverfahrens sprach sich die KBV für einen stärkeren Abbau der Doppelstrukturen in der Notfallversorgung aus (Eckpunkte der KBV und KV zur Weiterentwicklung der Notallversorgung 2017). Nach Ansicht der Bundesvereinigung werde somit auch ein Großteil der Notfallambulanzen obsolet und müsse geschlossen werden (Fricke 2017). Der Marburger Bund (MB) unterstützt den Gedanken ebenfalls, die ambulante und stationäre Notfallversorgung unter einem Dach zu organisieren (MB 2020). Bereits 2017 wurde dafür geworben, gemeinsame medizinische Anlaufstellen am Krankenhaus zu etablieren und so eine bessere Zusammenarbeit der Vertrags- und Krankenhausärzteschaft zu fördern (Konzeptpapier von KBV und Marburger Bund 2017). Die Deutsche Krankenhausgesellschaft (DKC) setzte sich vor dem Gesetzgebungsprozess für eine stärkere Verantwortung der Länder in der ambulanten Notfallversorgung ein und forderte, Krankenhäuser dauerhaft zur Teilnahme an der ambulanten Notfallversorgung zu ermächtigen. Die DKG lehnt daher die vorgeschlagenen Änderungen ab, die ambulante Notfallversorgung in INZ zu überführen. Zentralisierung von Leistungen führe nach Ansicht der DKC nur zu höheren Belastungen und Wartezeiten bei den Patientinnen und Patienten (DKG 2020). Aus Sicht des CKV-Spitzenverbandes ist eine gemeinsame Organisation der ambulanten und stationären Notfallversorgung nach bundeseinheitlichen Vorgaben an zentralen Standorten zu begrüßen. Die Patientin oder der Patient solle im Notfall eine bekannte, feste Anlaufstelle haben. Dort solle interdisziplinär ausgebildetes Personal entscheiden, in welcher Versorgungsebene die Patientin oder der Patient richtig aufgehoben ist (GKV-Spitzenverband 2019).

Die Analyse der Stellungnahmen zum Referentenentwurf zeigt, welche Herausforderungen, Gemeinsamkeiten sowie Konflikte bei den Reformbestrebungen bestehen. So gibt es etwa weiterhin unterschiedliche Auffassungen zwischen den Vertragsärzten und Krankenhäusern über Zuständigkeiten und Finanzierung der ambulanten Notfallversorgung. Die Krankenhäuser kritisieren, dass die Vergütung von Leistungen für Patienten, die in den Notaufnahmen der Kliniken nur ambulant behandelt werden, nicht wirtschaftlich sei (Haas et al. 2015). Die KBV argumentiert dagegen, dass ambulante Notfallpatientinnen und -patienten im Krankenhaus oder im INZ vornehmlich nur eine Steuerung in die richtige Versorgungsebene benötigen, aber es keiner Parallelstruktur zu den regulären Vertragsarztpraxen bedarf (KBV 2020). Aus einer übergeordneten Perspektive betrachtet bleibt festzustellen: Im Status quo werden die verschiedenen Standorte, Ressourcen und Akteure der ambulanten Notfallversorgung weder gemeinsam betrachtet noch nach einheitlichen Rahmenvorgaben unter Berücksichtigung des Versorgungsbedarfs geplant. Nachvollziehbare Kriterien, die definieren, wo eine durch Vertragsärzte betriebene Notdienstpraxis oder ein INZ am Krankenhaus sinnvoll ist bzw. wie die Zusammenarbeit mit den Krankenhäusern ausgestaltet werden soll, wird mit dem Referentenentwurf des BMG nun erstmals adressiert. 


\subsection{Lösungsansätze für eine Neustrukturierung}

Versteht man das Notfallstufenkonzept als Ausgangsbasis für die Neustrukturierung der gesamten Notfallversorgung, so sind in einem nächsten Schritt ergänzende, bundesweite Mindestvorgaben zu den vorzuhaltenden ambulanten Notfallstrukturen zu definieren. Der Referentenentwurf des BMG zur Reform der Notfallversorgung, der seit Januar 2020 vorliegt, greift diesen Gedanken auf. Demnach sollen an ausgewählten Krankenhäusern mit einer stationären Notfallstufe zukünftig Integrierte Notfallzentren (INZ) eingerichtet werden. Auch der Rettungsdienst soll eng auf das stationäre Notfallstufenkonzept abgestimmt werden, indem zukünftig nur noch Krankenhäuser angefahren werden sollen, welche die Anforderungen des Gemeinsamen Bundesausschusses für eine Teilnahme an einer Notfallstufe oder am Modul Notfallversorgung Kinder erfüllen. Eine entsprechende Regelung hatten die Krankenkassen ausdrücklich gefordert, um durch die direkte Anfahrt einer geeigneten Zielklinik unnötige Weiterverlegungen schwer erkrankter Patientinnen und Patienten zu vermeiden.

\subsubsection{Integrierte Notfallzentren (INZ)}

Kernstück des Referentenentwurfs ist die Einführung der Integrierten Notfallzentren. Diese Versorgungseinheit am Krankenhaus soll als zentrale, jederzeit zugängliche Einrichtung der medizinischen Akut- und Notfallversorgung an ausgewählten Krankenhausstandorten eingerichtet werden. Nach den Vorstellungen des Gesetzgebers soll sie von Patientinnen und Patienten als erste Anlaufstelle beansprucht werden. Bei Versorgungsbeginn soll im INZ eine qualifizierte und standardisierte Ersteinschätzung des Versorgungsbedarfs erfolgen und die aus medizinischer Sicht unmittelbar erforderliche ärztliche Versorgung geleistet oder in eine stationäre Versorgung überwiesen werden. Das INZ soll nach aktuellem Stand von KV und Krankenhaus gemeinsam, unter fachlicher Leitung der zuständigen KV betrieben werden. Um die organisatorische Verknüpfung zu den bestehenden Notaufnahmen zu gewährleisten und die bereits aufgebauten Portalpraxen in INZ zu überführen, sollen zwischen dem Krankenhaus und der zuständigen KV Kooperationsverträge geschlossen werden.

In den Überlegungen des SVR wird eine Verschmelzung aus Notaufnahme und ärztlichem Bereitschaftsdienst skizziert. Der Integrationsgrad ist allerdings unbestimmt, so könnte das INZ als „virtuelle Einheit“ klassifiziert werden, welche die Leistungen bei der KV oder dem Krankenhaus einkauft und nur eine Triage an einem gemeinsamen Tresen erbringt. Bei einer hundertprozentigen Integration besitzt das INZ eine eigene apparative Ausstattung und eigenes medizinisches Personal (Herr et al. 2020). Der Referentenentwurf bleibt in diesem Punkt unklar. Nach dem Entwurf wird das INZ als eigenständige Rechtspersönlichkeit definiert. Die KV kann grundsätzlich allerdings 
nicht selbst ärztliche Leistungen erbringen, sondern muss Ärzte bereitstellen, die im INZ tätig werden.

Um Qualitätsanforderungen sowie Vorgaben zum Leistungsumfang der INZ zu bestimmen, wird der G-BA mit einer neu zu erarbeitenden „Notfall-Richtlinie“ beauftragt. Dabei sollen Vorgaben zur personellen und apparativen Ausstattung von INZ definiert werden, auch um eine bedarfsgerechte Differenzierung der personellen Ausstattung nach Tages- und Nachtzeiten zu gewährleisten. Zusätzlich soll der G-BA das Verfahren zur qualifizierten Ersteinschätzung des Versorgungsbedarfs von Hilfesuchenden festlegen und den Umfang der im INZ zu erbringenden notdienstlichen Leistungen sowie die Sachverhalte der Weitervermittlung bundesweit einheitlich definieren. Es bleibt daher abzuwarten, ob das INZ zu einer eigenständigen und fachlich unabhängigen Versorgungseinheit ausgestaltet wird oder eine Lösung mit Fokus auf einen gemeinsamen Tresen von Krankenhaus und KV durch den G-BA erarbeitet wird, bei dem der oder die Hilfesuchende ausschließlich begutachtet und anschließend in die richtige Versorgungsebene überwiesen wird. Ergänzend sollte der G-BA mit der Entwicklung und Umsetzung eines Qualitätssicherungsverfahrens beauftragt werden, um das Versorgungsgeschehen transparent zu machen.

\subsubsection{Bedarfsplanung von INZ}

In Deutschland gibt es aktuell keine ausreichende Konzentration der Notfallversorgung. Hieran ändert auch das Konzept der stationären Notfallstufen nichts, da es lediglich die bestehenden Strukturen abbildet, jedoch weder den Versorgungsbedarf in einer Region berücksichtigt, noch eine Konzentration der Versorgung auf die höheren, gut ausgestatteten Notfallstufen fördert. In Bezug auf die Etablierung der INZ wurde seitens des BMC die Notwendigkeit einer am Versorgungsbedarf orientierten Planung und Konzentration der INZ-Standorte erkannt. Der Referentenentwurf sieht vor, dass der G-BA bundesweit einheitliche, bedarfsbezogene Planungsvorgaben zur Bestimmung der Anzahl und Standorte der INZ festlegen soll. Hierbei ist die Erreichbarkeit der INZ für die Bevölkerung einer Region ebenso entscheidend wie der Versorgungsbedarf einer Region. Die Festlegung der Anzahl und konkreten Standorte von INZ soll nach Maßgabe der Planungsvorgaben des G-BA durch Ausschüsse auf Landesebene erfolgen. Ausdrücklich zu begrüßen ist die gemeinsame Planung der Standorte der INZ in diesen Ausschüssen durch Kassenärztliche Vereinigungen, Landeskrankenhausgesellschaften und Krankenkassen auf Basis der bundesweit einheitlichen Planungskriterien. Es ist unerlässlich, die ambulante Notfallversorgung auf die Standorte zu konzentrieren, die dafür auch geeignet sind. Darüber hinaus ist eine Bedarfsplanung für die INZ dringend geboten, da einerseits eine flächendeckende wohnortnahe Erreichbarkeit gewährleistet sein muss und andererseits in Ballungsgebieten mit vielen stationären Notfallversorgern die INZ auf wenige, gut ausgestattete Standorte konzentriert werden sollten. 
Das RWI - Leibniz-Institut für Wirtschaftsforschung (RWI) hat im April 2018 einen Projektbericht zur Notfallversorgung im Auftrag der KBV veröffentlicht. Dabei wurde erstmals versucht, die Verteilung und Anzahl von Intersektoralen Notfallzentren in Deutschland zu modellieren. ${ }^{1}$ Für die Schätzung wurden zwei Bedingungen definiert: (1) Ambulante Notfallpatientinnen und -patienten sind maximal dazu bereit, eine Entfernung von 30 Minuten Fahrzeit zum nächstliegenden Versorger zu akzeptieren und (2) die Zentren werden an existierenden und bereits an der stationären Notfallversorgung teilnehmenden Krankhäusern angesiedelt. Im Ergebnis kann mit 736 Notfallzentren eine flächendeckende Erreichbarkeit (99,6\%) für die Bevölkerung gewährleistet werden. Eine weitere Konzentration der Notfallversorgung ist somit möglich, ohne die flächendeckende ambulante Notfallversorgung zu gefährden ( $\mathrm{Au}$ gurzky et al. 2018). Eine reine Betrachtung des Erreichbarkeitskriteriums greift jedoch zu kurz und vernachlässigt den bevölkerungsbezogenen Versorgungsbedarf. Nach Berechnungen der Krankenkassen würden in Berlin ca. vier INZ ausreichen, um eine vollumfängliche Abdeckung gemessen an der Erreichbarkeit innerhalb von $30 \mathrm{Pkw}$-Fahrzeitminuten zu gewährleisten. Ob in diesem Szenario auch der Versorgungsbedarf einer Millionenmetropole erfüllt werden kann, ist zu bezweifeln. Mit Blick auf die Planung ist es unerlässlich, auch den bevölkerungsbezogenen Versorgungsbedarf zu berücksichtigen. Für eine Umsetzung auf der Landesebene sind somit verbindliche Planungsinstrumente aber auch der Mut der Politik erforderlich, nicht alle stationären Notfallversorger mit einem INZ auszustatten.

\subsubsection{Reform des Rettungsdienstes}

Nach den Regelungsvorschlägen des Gesetzentwurfes zur Notfallreform soll die medizinische Notfallrettung Teil des Gesundheitswesens werden. Der Bereich teilt sich damit zukünftig in die medizinische Notfallrettung mit der vor Ort erbrachten medizinischen Leistung und Rettungsfahrten ins Krankenhaus. Beide Leistungen können unabhängig voneinander erbracht und abgerechnet werden. Damit ist es nicht mehr erforderlich, dass die Patientin oder der Patient zur Abrechenbarkeit eines Rettungseinsatzes ins Krankenhaus gefahren wird. Die Fahrt ins Krankenhaus erübrigt sich, wenn sich nach Einschätzung von Notfallsanitäterinnen oder Notfallsanitätern bzw. Notärztin oder Notarzt herausstellt, dass die Patientin oder der Patient in häuslicher Umgebung ausreichend versorgt werden kann. Das war eine überfällige Neuregelung, die nicht unerheblich dazu beitragen könnte, dass Notaufnahmen entlastet und unnötige Krankenhausaufenthalte vermieden werden können.

1 Das Notfallzentrum ähnelt dem Konzept des SVR, es soll einen zentralen Anlaufpunkt für alle Notfalpatientinnen und -patienten in einer Region etabliert werden. Idealerweise befindet sich ein INZ direkt im oder in der Nähe eines Krankenhauses (Beivers 2018). 


\subsubsection{Gemeinsames Notfallleitsystem}

Das im Referentenentwurf beschriebene gemeinsame Notfallleitsystem (GNL) soll als eine digital unterstützte, verbindliche Kooperation zwischen den Trägern der Rettungsleitstellen der Rufnummer 112 und den KVen ausgestaltet werden. Es soll Versicherte in medizinischen Notsituationen auf Basis einer standardisierten Ersteinschätzung in die geeignete Versorgungsstruktur lotsen. Dabei sind die Zuständigkeiten im Gesetzentwurf klar geregelt: Bei lebensbedrohlichen Notfällen ist die Rufnummer 112 zuständig, die Leistungen der medizinischen Notfallrettung und Krankentransporte disponiert. Die notdienstliche Versorgung für nicht lebensbedrohliche Notfälle wird über die Rufnummer 116117 disponiert. Durch einen Echtzeitdatenaustausch soll die Zusammenarbeit zwischen GNL, Krankenhäusern, Rettungsdienst und KV-Bereitschaftsdienst verbessert werden. Der G-BA soll hierfür die Kooperationsverpflichtungen definieren und Vorgaben zur digitalen Vernetzung schaffen. Weiterhin soll er die Regelungskompetenz für eine bundeseinheitliche Datenerfassung der medizinischen Notfallversorgung erhalten.

Die Aufgabenzuweisung an den G-BA ist folgerichtig, um einen bundeseinheitlichen Rahmen für die GNL zu schaffen. Ziel muss darüber hinaus auch die Entwicklung und Implementierung eines bundeseinheitlichen Ersteinschätzungsverfahrens sein. Die Digitalisierungsoffensive des Gesetzentwurfs ist überfällig und daher uneingeschränkt zu begrüßen. Wenn die Rettungsdienste zukünftig in Echtzeit einen Überblick über die aufnahmebereiten Notfallkrankenhäuser haben, können Patientinnen und Patienten schneller in eine geeignete Versorgungsstruktur gebracht werden, da die Zeit für telefonische Einzelanfragen bei den Krankenhäusern entfällt. Allerdings muss die länderübergreifende Interoperabilität der eingesetzten Softwaresysteme gewährleitet sein, damit zukünftig weder die Kommunikation noch die Rettungswagen an der Landesgrenze Halt machen. Angesichts der fehlenden Grundgesetzänderung, die dafür gesorgt hätte, dass auch die Rettungsleitstellen vom Bereich der Gefahrenabwehr in den Regelungsbereich des Gesundheitswesens gewechselt wären, wird die Teilnahme der 112-Leitstellen am GNL nur freiwillig erfolgen können. Dadurch wird es leider deutlich erschwert, eine insbesondere aus Patientensicht gebotene bundesweit einheitliche Struktur für die GNL zu schaffen. An dieser Stelle bleibt die Reform des Rettungswesens hinter dem Notwendigen zurück.

\subsubsection{Auswahl der geeigneten Versorgungsebene}

Jeder Patient und jede Patientin, der bzw. die sich mit einem Hilfegesuch an das GNL wendet, soll zukünftig ein standardisiertes Ersteinschätzungsverfahren durchlaufen, um den individuellen Hilfebedarf sowie die geeignete Versorgungsebene zu ermitteln. Die Entscheidung des GNL auf Basis dieses Verfahrens wird einer ärztlichen Verordnung gleichgestellt, d.h. Rettungs- 
fahrten und Krankentransporte können ohne gesonderte ärztliche Verordnung disponiert werden. Die zur Weiterbehandlung erforderlichen Informationen im Rahmen der Notfallrettung werden vom GNL an das anzufahrende Integrierte Notfallzentrum (INZ) oder Krankenhaus digital übermittelt. Ist auf Basis der Ersteinschätzung eine stationäre Behandlung voraussichtlich nicht erforderlich, ist das nächsterreichbare INZ anzufahren. Scheint hingegen eine stationäre Aufnahme notwendig, sind grundsätzlich nur Krankenhäuser anzufahren, die gemäß den Mindestanforderungen des G-BA an der Notfallversorgung teilnehmen. Der G-BA soll darüber hinaus festlegen, in welchen Fällen im Sinne einer zielgerichteten Behandlung anstelle des nächsterreichbaren Notfallkrankenhauses ein spezialisiertes Krankenhaus anzufahren ist. Eine solche Regelung wurde seitens der Krankenkassen schon länger gefordert, da nur Krankenhäuser, welche die Mindestanforderungen des G-BA erfüllen, geeignete Strukturen (z.B. Intensivbetten, CT) zur Versorgung von stationär behandlungsbedürftigen Notfallpatientinnen und -patienten vorhalten. Erfolgreiche und bereits etablierte Projekte zur Echtzeitdatenübertragung, wie z.B. IVENA, enden heute oft an den Kreis- und Landesgrenzen. Insbesondere der Auftrag an den G-BA, zu definieren, welche Krankenhäuser für die Behandlung der Tracerdiagnosen geeignet sind, stellt eine wesentliche Verbesserung im Sinne der Patientinnen und Patienten dar. Bei schweren Notfällen wie Polytrauma oder Herzinfarkt ist es entscheidend für den Behandlungserfolg, die Patientinnen und Patienten sofort in ein für ihren Behandlungsfall geeignetes Krankenhaus zu bringen. Hier ist die Verbindung des CNL zum stationären Notfallstufensystem gut gelungen.

\subsection{Fazit}

Der vorliegende Referentenentwurf für ein Gesetz zur Reform der Notfallversorgung liefert positive Impulse zur Vereinheitlichung der bisher regional stark unterschiedlich entwickelten Notfallversorgung. Die umfassenden Strukturverbesserungen brauchen einen mutigen Gestaltungswillen des Gesetzgebers, denn eine patientenorientierte Notfallversorgung erfordert gemeinsame Verantwortung und Gestaltung von Kassenärztlicher Vereinigung, Krankenhausgesellschaften und Krankenkassen. Insgesamt erscheinen die ersten Überlegungen geeignet, um die derzeit sowohl für die Patientinnen und Patienten als auch das Klinikpersonal unbefriedigende Situation in den Krankenhausnotaufnahmen grundlegend zu verändern. Die systematische Etablierung von INZ an den vom G-BA normierten stationären Notfallversorgern in Verbindung mit klar geregelten Zuständigkeiten, bundeseinheitlichen Anforderungen an die Angebotsstrukturen, den aufsuchenden Bereitschaftsdienst und den telemedizinischen Bereitschaftsdienst sollten dazu führen, dass die bisherige Fehlinanspruchnahme der Notaufnahmen durch Patientinnen und Patienten mit originär ambulantem Versorgungsbedarf zukünftig vermieden wird. Andere europäische Länder haben bereits bewiesen, dass dies 
funktionieren kann. Es bedarf daher nur noch des versorgungspolitischen Willens für ein sektorenübergreifendes Vorgehen.

\section{Take home messages}

- Mit dem gestuften System von Notfallstrukturen in Krankenhäusern hat der Gemeinsame Bundesausschuss erstmals Mindestanforderungen für die Vorhaltung von Notfallstrukturen in der stationären Versorgung adressiert.

- Aus Patientenperspektive trägt die gegenwärtige fehlende Abstimmung zwischen vertragsärztlichem Bereitschaftsdienst, Rettungsdienst und Notaufnahmen der Krankenhäuser dazu bei, dass Patientinnen und Patienten nicht immer in der angemessenen Versorgungsebene behandelt werden.

- Die Reformüberlegungen des Gesetzgebers zur Neuordnung der Notfallversorgung liefern positive Impulse zur Vereinheitlichung der bisher regional stark unterschiedlich entwickelten Notfallversorgung.

- Die systematische Etablierung von Integrierten Notfallzentren an den vom Gemeinsamen Bundesausschuss normierten stationären Notfallstrukturen kann zu einer bundeseinheitlichen und hochwertigen Angebotsstruktur führen und die bisherige Fehlinanspruchnahme der Notfallaufnahmen durch Patientinnen und Patienten mit originär ambulanten Versorgungsbedarf vermeiden.

- Der beabsichtigte Aufbau von gemeinsamen Notfallleitsystemen mit verbindlichen Kooperationsverpflichtungen und Vorgaben zur Vernetzung sind ein wichtiger Schritt für die Digitalisierungsoffensive des Rettungswesens. Aus Patientensicht kann so eine schnellere Ersteinschätzung erfolgen und die Zuweisung in die geeignete Versorgungsstruktur gelingen.

\section{Literatur}

AOK BV (2020) Stellungnahme des AOK-Bundesverbandes zur Fachanhörung des BMG am 17.02.2020. URL: https://aok-bv.de/imperia/md/aokbv/positionen/stellungnahmen/stellungnahme_refe_notfallversorung.pdf (abgerufen am 08.06.2020)

Augurzky B, Beivers A, Breidenbach P, Budde R, Emde A, Haering A, Kaeding M, Roßbach-Wilk E, Straub N (2018) Notfallversorgung in Deutschland. Projektbericht im Auftrag der Kassenärztlichen Bundesvereinigung, RWI - Leibniz-Institut für Wirtschaftsforschung (Hrsg.), Projektberichte. Essen.

Baier N, Geissler A, Bech M, Berstein D, Cowling TE, Jackson T, van Manen J, Rudkjobing A Quentin W (2019) Emergency Emergency and urgent care systems in Australia, Denmark, England, France, Germany and the Netherlands - Analyzing organization, payment and reforms. Health Policy 123(11), 1-10

Beivers A (2018) Neujustierung der Notfallversorgung durch sektorenübergreifende Notfallzentren. In: Pfannstiel MA, Da-Cruz P, Rasche C (Hrsg.) Entrepreneurship im Gesundheitswesen: Digitalisierung, Innovationen, Gesundheitsversorgung, Band III. 253-267. Springer Verlag Berlin

BMG - Bundesministeriums für Gesundheit (2020) Entwurf eines Gesetzes zur Reform der Notfallversorgung. URL: https://www.bundesgesundheitsministerium.de/fileadmin/Dateien/3_Downloads/Gesetze_und_Verordnungen/GuV/N/Referentenentwurf_zur_Reform_der_Notfallversorgung.pdf (abgerufen am 08.06.2020) 
DKG - Deutsche Krankenhausgesellschaft (2020) Stellungnahme der Deutschen Krankenhausgesellschaft zum Referentenentwurf eines Gesetzes zur Reform der Notfallversorgung (Stand: 10. Februar 2020). URL: https://www.dkgev.de/fileadmin/default/Mediapool/1_DKG/1.3_Politik/Stellungnahmen/2020-02-10_ DKG-Stellungnahme_Referentenentwurf_Gesetz_zur_Reform_der_Notfallversorgung.pdf (abgerufen am 08.06.2020)

Dräther H, Schäfer T (2017) Die ambulante Notfallversorgung in Notfallambulanzen und bei Vertragsärzten im Zeitraum 2009 bis 2014. In: Klauber |, Geraedts M, Friedrich J, Wasem I. (Hrsg.) Krankenhaus-Report 2017. 25-40. Schattauer Stuttgart.

Fischer M, Kehrberger E, Marung H, Moecke H, Prückner S, Trentzsch H, Urban B (2016) Eckpunktepapier 2016 zur notfallmedizinischen Versorgung der Bevölkerung in der Prähospitalphase und in der Klinik. Notfall + Rettungsmedizin 19, 387-395

Fricke A (2017) KBV-Chef Gassen - Hunderte Klinik-Ambulanzen überflüssig. URL: https://www.aerztezeitung.de/ Politik/KBV-Chef-Gassen-Hunderte-Klinik-Ambulanzen-ueberfluessig-299460.html (abgerufen am 08.06.2020).

Gemeinsamer Bundesausschuss (2018) Anlage 1 zu den Tragenden Gründen zum Beschluss des Gemeinsamen Bundesausschusses über die Erstfassung der Regelungen zu einem gestuften System von Notfallstrukturen in Krankenhäusern gemäß $\$ 136 c$ Absatz 4 SGB V vom 19. April 2018. URL: https://www.g-ba.de/ downloads/40-268-5393/2018-04-19_Not-Kra-R_TrG_Anlage.pdf (abgerufen am 19.11.2019)

Gesundheitsberichterstattung des Bundes (1998) Gesundheitsbericht für Deutschland, 1998. Rettungsdienste und Krankentransportwesen, Kapitel 6.13. URL: www.gbe-bund.de/gbe10/abrechnung.prc_abr_test_logon?p_uid=gast\&p_aid=0\&p_knoten=FID\&p_sprache=D\&p_suchstring=1137::Gesundheitsausgaben (Stand 19.05.2020)

GKV-Spitzenverband (2019) Die Notfallversorgung von morgen - ein Ausblick. URL: https://www.gkv-spitzenverband.de/media/dokumente/presse/pressekonferenzen_gespraeche/2019_2/2019-08-28_Vortrag_Notfallversorgung.pdf (abgerufen am 10.06.2020)

Haas C, Larbig M, Schöpke T, Lübke-Naberhaus, K-D, Schmidt C, Brachmann M, Dodt C (2015) Gutachten zur ambulanten Notfallversorgung im Krankenhaus - Fallkostenkalkulation und Strukturanalyse der Management Consult Kestermann GmbH (MCK) erstellt in Kooperation mit der Deutschen Gesellschaft interdisziplinäre Notfall- und Akutmedizin e.V. (DGINA). Management Consult Kestermann. URL: https://www. dkgev.de/fileadmin/default/Mediapool/2_Themen/2.2_Finanzierung_und_Leistungskataloge/2.2.4. Ambulante_Verguetung/2.2.4.4._Ambulante_Notfallvehandlung_durch_Krankenhaeuser/2015-02-17_ Gutachten_zur_ambulanten_Notfallversorgung_im_Krankenhaus_2015.pdf (abgerufen am 07.06.2020).

Hermann I (2019) Herrmann kritisiert Reformpläne des Bundes im Notfallwesen. URL: https://www.bayern.de/ herrmann-kritisiert-reformplaene-des-bundes-im-notfallwesen/ (abgerufen am 07.06.2020)

Herr D, Schreyögg I, Gerlach FM (2019) Integrierte Notfallzentren - Kernelement der zukünftigen Notfallversorgung. Gesundheit und Sozialpolitik 73(4-5), 44-49

KBV - Kassenärztliche Bundesvereinigung (2020) Stellungnahme der KBV zum Referentenentwurf des Bundesministeriums für Gesundheit vom 16. Dezember 2019. URL: https://www.kbv.de/media/sp/KBV-Stellungnahme_Referentenentwurf_Notfallreform.pdf (abgerufen am 07.06.2020)

KBV, Marburger Bund (2017) Konzeptpapier von KBV und Marburger Bund 2017: Integrative Notfallversorgung aus ärztlicher Sicht - Konzeptpapier von KBV und Marburger Bund vom 18.09.2017. URL: https://www. marburger-bund.de/sites/default/files/files/2018-07/17-9-18-mb-kbv-konzeptpapier-notfallversorgung. pdf (abgerufen am 07.06.2017)

KBV und KV zur Weiterentwicklung der ambulanten Notfallversorgung (2017) Eckpunkte der Kassenärztlichen Bundesvereinigung und der Kassenärztlichen Vereinigungen zur Weiterentwicklung der ambulanten Notfallversorgung vom 29.03.2017. URL: https://www.kbv.de/media/sp/2017-03-29_Eckpunkte_Weiterentwicklung_ambulante_Notfallversorgung.pdf (abgerufen am 07.06.20202)

MB - Marburger Bund (2020) Stellungnahme des Marburger Bund Bundesverbandes zu dem Referentenentwurf des Bundesministeriums für Gesundheit zum Entwurf eines Gesetzes zur Reform der Notfallversorgung (Stand: 08.01.2020). URL: https://www.marburger-bund.de/sites/default/files/files/2020-02/ MB-Stellungn_RefEntwurf_Notfallversorgung_07022020.pdf (abgerufen am 07.06.2020)

Sachverständigenrat zur Begutachtung für die Konzertierte Aktion im Gesundheitswesen (SVR) (2017) Weitentwicklung der Notfallversorgung in Deutschland. URL: http://www.svr-gesundheit.de/fileadmin/user_upload/2017-09-08_Notfall_Webseite.pdf (abgerufen am 07.06.2020) 
Slowik M, Malzahn I (2017) Erste Hilfe für den Notfall. Gesundheit und Gesellschaft 20(10), 23-27

Slowik M, Wehner C, Dräther H, Fahlenbrach C, Richard S (2018) Sektorübergreifende Neuordnung der Notfallversorgung. In: Klauber I, Geraedts M, Friedrich J, Wasem J. (Hrsg.) Krankenhaus-Report 2018. 233-255. Schattauer Stuttgart

Stelle zur trägerübergreifenden Qualitätssicherung im Rettungsdienst Baden-Württemberg (SQRBW). 2017: Qualitätsbericht des Rettungsdienstes Baden-Württemberg für das Jahr 2016 vom September 2017. URL: https://www.sqrbw.de/de/sqr-bw/qualitaetsberichte (abgerufen am 12.06.2020)

Wikipedia (2020) Liste der BOS-Leitstellen. URL: https://de.wikipedia.org/wiki/Liste_der_BOS-Leitstellen (abgerufen am 13.05.2020)

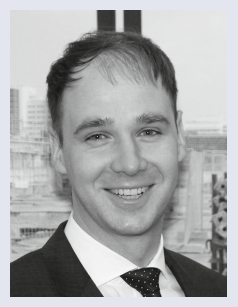

Michael Slowik, M.Sc. Gesundheitsökonomie

Michael Slowik ist Gesundheitsökonom und seit Mai 2016 als Referent für Krankenhausvergütung beim AOK-Bundesverband in Berlin tätig. Aktuell beschäftigt sich Herr Slowik mit der Finanzierung der Pflegebudgets im Krankenhaus und weiterer Vergütungssysteme. Zusätzlich erarbeitete er Reformideen zur Neustrukturierung der Notfallversorgung für die AOK-Gemeinschaft. Zuvor war er beim IGES Institut und an der School of Public Health in Michigan, USA. Interessenschwerpunkte sind vor allem innovative Vergütungsmethoden, Ordnungspolitik und die Strukturreform der Krankenhausversorgung.



\section{Kerstin Bockhorst, M.Sc. Public Health}

Kerstin Bockhorst ist Gesundheitswissenschaftlerin und seit Juli 2016 Referentin für Krankenhausvergütung beim GKV-Spitzenverband in Berlin. Dort befasst sie sich u.a. mit der Sicherstellung der Krankenhausversorgung in dünn besiedelten Regionen und der Neustrukturierung der Notfallversorgung in Deutschland. Zuvor war sie Referatsleiterin für Krankenhausfinanzierung beim Verband der Ersatzkassen (vdek) e.V. in der Verbandszentrale in Berlin. Von 2012-2014 war sie wissenschaftliche Mitarbeiterin für Versorgungsforschung bei der TMF - Technologie- und Methodenplattform für die vernetzte medizinische Forschung e.V. Zuvor war sie als wissenschaftliche Mitarbeiterin in der Universitätsmedizin Göttingen und als Projektmanagerin im Herz- und Diabeteszentrum NRW tätig. 